\title{
Optic Perineuritis Associated with Nivolumab Treatment for Non-Small Cell Lung Cancer
}

\author{
Kenji Takada Keiichi Fujiwara Eri Ando Kiriko Onishi \\ Tadahiro Kuribayashi Sho Mitsumune Yuki Takigawa \\ Hiroaki Matsuura Hiromi Watanabe Kenichiro Kudo \\ Akiko Sato Ken Sato Takuo Shibayama \\ Department of Respiratory Medicine, National Hospital Organization Okayama Medical \\ Center, Okayama, Japan
}

\section{Keywords}

Optic perineuritis · Immune checkpoint inhibitor · Immune-related adverse events .

Nivolumab

\begin{abstract}
We report the case of a 54-year-old man who was treated with nivolumab for recurrent squamous cell lung cancer. After 7 cycles of nivolumab treatment, the patient presented to our hospital with right eye vision loss. Gadolinium-enhanced magnetic resonance imaging of the brain showed enhancement around the optic nerve sheath. This finding and his symptoms led to the diagnosis of optic perineuritis (OPN). Steroid pulse therapy was administered twice although there was no remarkable improvement in his visual field defect. The relationship between OPN and nivolumab is unclear. However, immune-related adverse events caused by immune checkpoint inhibitors should be considered.
\end{abstract}

(C) 2021 The Author(s).

Published by S. Karger AG, Basel

\section{Introduction}

Nivolumab is a human monoclonal antibody against programmed cell death 1 with antitumor effects on lung cancer [1]. The increasing use of nivolumab and other immune checkpoint inhibitors (ICIs) in cancer treatment has led to an increase in immune-related adverse events (irAEs). Skin, gastrointestinal, and endocrine irAEs are often reported. However, optic neuritis frequency is low, and its clinical features are unknown [2-4]. We report a case of optic perineuritis (OPN) during nivolumab therapy for squamous cell lung cancer, which was challenging to treat due to steroid resistance. 


\section{Case Report}

A 54-year-old man was diagnosed with squamous cell lung cancer. He underwent right upper lobectomy and concomitant chest wall resection in July 2017. Following the surgery, he received thoracic radiotherapy (RT) for the residual lesion in the intervertebral foramen of the fourth thoracic vertebra (total 60 Gy per 30 fractions). Two months after thoracic RT, multiple pulmonary metastases were observed in the bilateral lung field. He was initially treated with combination chemotherapy comprising carboplatin and nab-paclitaxel although his disease progressed after 2 chemotherapy cycles. As second-line chemotherapy, nivolumab (at a dose of $3 \mathrm{mg} / \mathrm{kg}$, biweekly) was administered in April 2018. During nivolumab treatment, muscle metastasis in the neck occurred with pain. Additional RT achieved tumor shrinkage. Computed tomography scans also showed a reduction in the size of lung metastases. Therefore, we continued nivolumab treatment.

After the seventh dose of nivolumab, visual loss in the right eye was noted in July 2018. An ophthalmologist performed visual acuity test and Goldmann perimetry. His right visual acuity declined to 20/2000, and an inferior nasal visual field defect was observed (Fig. 1). Gadolinium-enhanced cranial magnetic resonance imaging showed enhancement around the optic nerve sheath (Fig. 2). There were no abnormal findings in the brain, optic disc, or retina. Based on the radiological findings and his symptoms, the patient was diagnosed with OPN.

Laboratory findings revealed slightly elevated C-reactive protein level at $0.48 \mathrm{mg} / \mathrm{dL}$, without leukocytosis (Table 1). Serologic examination revealed a negative rheumatoid factor and antinuclear antibody test results. In addition, double-stranded deoxyribonucleic acidspecific antibody, anti-cyclic citrullinated peptide antibody, anti-neutrophil cytoplasmic antibody, and immunoglobulin G4 levels were not elevated. Further, anti-aquaporin-4 antibody, which is recognized in patients with optic neuromyelitis, was also negative. Examination of the cerebrospinal fluid was not conducted because of the patient's refusal.

OPN was likely caused by nivolumab. Thus, we discontinued further nivolumab administration. Instead, we initiated steroid pulse therapy (intravenous methylprednisolone 1,000 mg/ day for 3 days), followed by oral prednisolone (30 mg). Despite these therapies, his visual acuity did not improve, and there was no remarkable change in his visual field defect. A second steroid pulse therapy was administered although the effect was temporary. We considered administering immunosuppressants against OPN although his lung cancer progressed rapidly. He did not want to receive further anticancer treatment and requested palliative care. We subsequently reduced the steroid dose to oral prednisolone $(15 \mathrm{mg}$ ) because of the poor efficacy of OPN. He was transferred to a palliative care unit at another hospital.

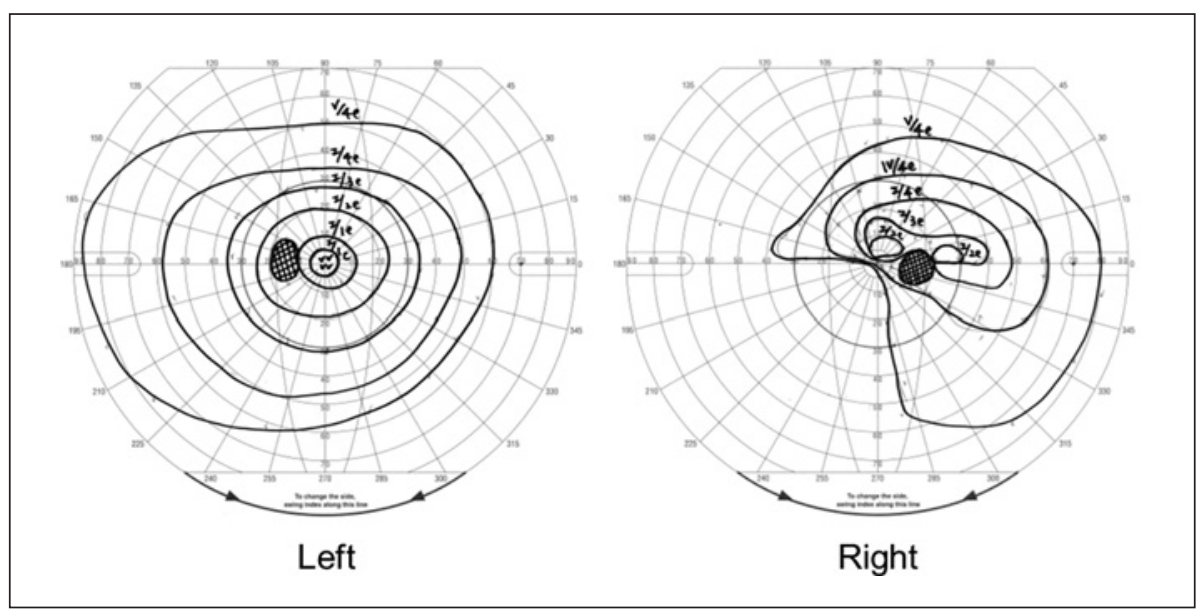

Fig. 1. Goldmann perimetry showing an inferior nasal visual field defect in the right eye.

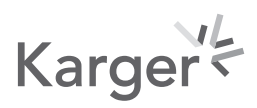


Fig. 2. Head gadolinium-enhanced, fatsaturated T2 MRI. a In the axial view, the "tram-tracks" sign of the optic nerve sheath is observed. b In the coronal view, the "doughnut" sign of the optic nerve sheath is observed. MRI, magnetic resonance imaging.
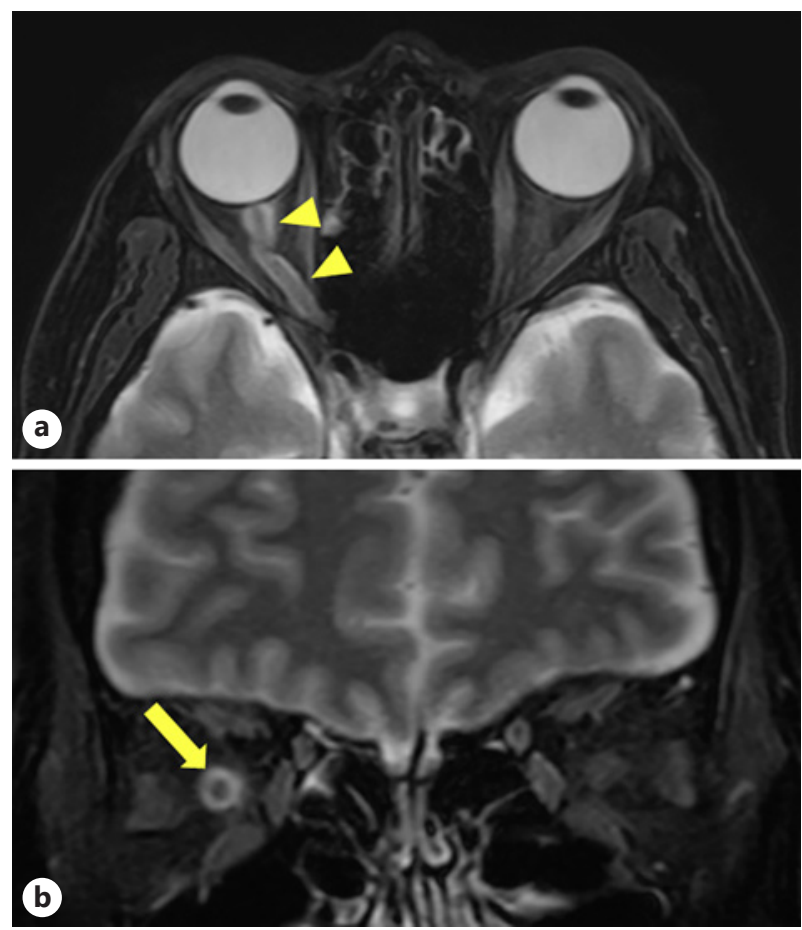

Table 1. Laboratory findings at the onset of OPN

\begin{tabular}{|c|c|c|c|c|c|c|c|c|}
\hline \multicolumn{3}{|c|}{ Hematology } & \multirow{2}{*}{$\begin{array}{l}\text { ALT } \\
\text { LDH }\end{array}$} & \multirow{2}{*}{$\begin{array}{l}10 \\
339\end{array}$} & \multirow{2}{*}{$\begin{array}{l}\mathrm{U} / \mathrm{L} \\
\mathrm{U} / \mathrm{L}\end{array}$} & \multirow{2}{*}{$\begin{array}{l}\text { CEA } \\
\text { CA19-9 }\end{array}$} & \multirow{2}{*}{$\begin{array}{l}7.6 \\
0.5\end{array}$} & \multirow{2}{*}{$\begin{array}{l}\mathrm{ng} / \mathrm{mL} \\
\mathrm{U} / \mathrm{mL}\end{array}$} \\
\hline WBC & 8,500 & $/ \mu \mathrm{L}$ & & & & & & \\
\hline Seg & 83.0 & $\%$ & ALP & 209 & $\mathrm{U} / \mathrm{L}$ & CYFRA & $(+)$ & $\mathrm{ng} / \mathrm{mL}$ \\
\hline Mon & 6.0 & $\%$ & $\gamma$-GTP & 63 & $\mathrm{U} / \mathrm{L}$ & $\operatorname{IgG}$ & 1,263 & $\mathrm{mg} / \mathrm{dL}$ \\
\hline Lym & 9.3 & $\%$ & $\mathrm{TP}$ & 7.2 & $\mathrm{~g} / \mathrm{dL}$ & $\operatorname{Ig} A$ & 331 & $\mathrm{mg} / \mathrm{dL}$ \\
\hline Eos & 1.2 & $\%$ & ALB & 4.1 & $\mathrm{~g} / \mathrm{dL}$ & $\operatorname{IgM}$ & 109 & $\mathrm{mg} / \mathrm{dL}$ \\
\hline Bas & 0.5 & $\%$ & CRE & 0.54 & $\mathrm{mg} / \mathrm{dL}$ & $\operatorname{IgE}$ & 881.0 & $\mathrm{IU} / \mathrm{mL}$ \\
\hline RBC & 413 & $\times 10^{4} / \mu \mathrm{L}$ & BUN & 17 & $\mathrm{mg} / \mathrm{dL}$ & $\operatorname{IgG} 4$ & 45.0 & $\mathrm{mg} / \mathrm{dL}$ \\
\hline $\mathrm{Hgb}$ & 12.8 & $\mathrm{~g} / \mathrm{dL}$ & $\mathrm{Na}$ & 137 & $\mathrm{mEq} / \mathrm{L}$ & $\mathrm{RF}$ & 11 & $\mathrm{U} / \mathrm{mL}$ \\
\hline Hct & 37.9 & $\%$ & $\mathrm{~K}$ & 4.4 & $\mathrm{mEq} / \mathrm{L}$ & ANA & $<\times 40$ & \\
\hline PLT & 31.1 & $\times 10^{4} / \mu \mathrm{L}$ & $\mathrm{Cl}$ & 103 & $\mathrm{mEq} / \mathrm{L}$ & PR3-ANCA & $<0.1$ & $\mathrm{U} / \mathrm{mL}$ \\
\hline ESR & 37 & $\mathrm{~mm} / \mathrm{h}$ & Serology & & & MPO-ANCA & $<1.0$ & $\mathrm{U} / \mathrm{mL}$ \\
\hline \multicolumn{3}{|c|}{ Biochemistry } & CRP & 0.48 & $\mathrm{mg} / \mathrm{dL}$ & Anti-CCP Ab & 0.9 & $\mathrm{U} / \mathrm{mL}$ \\
\hline T-Bil & 0.3 & $\mathrm{mg} / \mathrm{dL}$ & $\mathrm{HbA1c}$ & 6.0 & $\mathrm{mg} / \mathrm{dL}$ & Anti-dsDNA Ab & $<10$ & $\mathrm{IU} / \mathrm{mL}$ \\
\hline AST & 10 & $\mathrm{U} / \mathrm{L}$ & KL-6 & 322 & $\mathrm{U} / \mathrm{mL}$ & Anti-AQP4 Ab & $<1.5$ & $\mathrm{U} / \mathrm{mL}$ \\
\hline
\end{tabular}

WBC, white blood cell; RBC, red blood cell; Hgb, hemoglobin; Hct, hematocrit; PLT, platelet; ESR, erythrocyte sedimentation rate; T-Bil, total bilirubin; AST, aspartate aminotransferase; ALT, alanine aminotransferase; LDH, lactate dehydrogenase; ALP, alkaline phosphatase; TP, total protein; ALB, albumin; CRE, creatinine; BUN, blood urea nitrogen; CRP, C-reactive protein; HbA1c, hemoglobin A1c; KL-6, Krebs von den lungen-6; CEA, carcinoembryonic antigen; CA19-9, carbohydrate antigen 19-9; CYFRA, cytokeratin 19 fragment; IgG, immunoglobulin G; IgA, immunoglobulin A; IgM, immunoglobulin M; IgE, immunoglobulin E; IgG4, immunoglobulin 4; RF, rheumatoid factor; ANA, antinuclear antibody; PR3-ANCA, proteinase 3-anti-neutrophil cytoplasmic antibody; MPO-ANCA, myeloperoxidase-anti-neutrophil cytoplasmic antibody; Anti-CCP ab, anti-cyclic citrullinated peptide antibody; Anti-dsDNA ab, anti-double-stranded DNA antibody; Anti-AQP4 Ab, anti-aquaporin 4 antibody; OPN, optic perineuritis. 


\section{Discussion}

OPN is an inflammatory disease that causes inflammation of the optic nerve sheath, resulting in eye pain and visual loss in most patients [5, 6]. Gadolinium-enhanced cranial MRI, as well as visual acuity and field examinations, is useful to diagnose OPN. Typically, the optic nerve sheath is enhanced. It exhibits the tram-tracks sign on axial view and doughnut sign on a coronal view [5, 7]. The present case involved visual loss, and the tramtrack and doughnut signs were observed on enhanced MRI. Thus, the patient was diagnosed with OPN.

OPN is divided into 2 categories, idiopathic and secondary, depending on the cause of the disease. The causes of secondary OPN include sarcoidosis [8], immunoglobulin G4-related disease [6], Behcet's disease [9], vasculitis [10], infection [11], and neoplasms [12, 13]. There was no evidence of infectious disease or autoimmune disease in the laboratory findings in the present case, as shown in Table 1. Malignancies aside from the preexisting lung cancer were not detected on computed tomography scans and cranial MRI.

It is unclear whether the present case was idiopathic. The outcome of steroid treatment is dependent on the time between the appearance of symptoms and initiation of treatment $[5,11]$. The idiopathic group reportedly had a better convalescence than the second group [14]. In the present case, steroid administration had little effect on the patient's symptoms despite prompt treatment initiation. Considering his medical history, particularly nivolumab treatment, we considered OPN as an irAE rather than idiopathic.

Neurologic irAEs are less common than skin, gastrointestinal, or endocrine irAEs. The frequently observed orbital adverse events of ICIs include uveitis and eye dryness. However, optic neuritis has been associated with ICIs [15-17]. To the best of our knowledge, no reports on OPN as an irAE have been published. Although OPN is a rare irAE, it can cause irreversible visual loss despite appropriate treatment, as in our case. Thus, this irAE should be considered when prescribing ICIs because OPN negatively affects the patients' quality of life.

\section{Statement of Ethics}

Written informed consent for publication of this case report and any accompanying images was obtained from the patient's wife.

\section{Conflict of Interest Statement}

The authors state that they have no conflicts of interest.

\section{Funding Sources}

We declare that the authors received no funds for this case report.

\section{Author Contributions}

All the authors contributed to this manuscript and approved the final version.

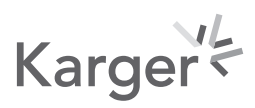




\section{References}

1 Borghaei H, Paz-Ares L, Horn L, Spigel DR, Steins M, Ready NE, et al. Nivolumab versus docetaxel in advanced nonsquamous non-small-cell lung cancer. N Engl J Med. 2015;373(17):1627-39.

2 Hodi FS, O’Day SJ, McDermott DF, Weber RW, Sosman JA, Haanen JB, et al. Improved survival with ipilimumab in patients with metastatic melanoma. N Engl J Med. 2010;363:711-23.

3 Robert C, Thomas L, Bondarenko I, O’Day S, Weber J, Garbe C, et al. Ipilimumab plus dacarbazine for previously untreated metastatic melanoma. N Engl J Med. 2011;364:2517-26.

4 Cuzzubbo S, Javeri F, Tissier M, Roumi A, Barlog C, Doridam J, et al. Neurological adverse events associated with immune checkpoint inhibitors: review of the literature. Eur J Cancer. 2017;73:1-8.

5 Purvin V, Kawasaki A, Jacobson DM. Optic perineuritis: clinical and radiographic features. Arch Ophthalmol. 2001;119:1299-306.

6 Hickman SJ. Optic perineuritis. Curr Neurol Neurosci Rep. 2016;16(2):16.

7 Pakdaman MN, Sepahdari AR, Elkhamary SM. Orbital inflammatory disease: pictorial review and differential diagnosis. World J Radiol. 2014;6:106-15.

8 Yu-Wai-Man P, Crompton DE, Graham JY, Black FM, Dayan MR. Optic perineuritis as a rare initial presentation of sarcoidosis. Clin Exp Ophthalmol. 2007;35:682-4.

9 Lai C, Sun Y, Wang J, Purvin VA, He Y, Yang Q, et al. Optic perineuritis in Behçet disease. J Neuroophthalmol. 2015;35:342-7.

10 Takazawa T, Ikeda K, Nagaoka T, Hirayama T, Yamamoto T, Yanagihashi M, et al. Wegener granulomatosisassociated optic perineuritis. Orbit. 2014;33:13-6.

11 Bergman 0, Andersson T, Zetterberg M. Optic perineuritis: a retrospective case series. Int Med Case Rep J. 2017;10:181-8.

12 Dutton JJ, Anderson RL. Idiopathic inflammatory perioptic neuritis simulating optic nerve sheath meningioma. Am J Ophthalmol. 1985;100:424-30.

13 Townsend JH, Dubovy SR, Pasol J, Lam BL. Transient optic perineuritis as the initial presentation of central nervous system involvement by pre-B cell lymphocytic leukemia. J Neuroophthalmol. 2013;33:162-4.

14 Takemaru M, Tachiyama K, Shiga Y, Kanaya Y, Shimoe Y, Kuriyama M. A case of optic perineuritis-A literature review of Japanese cases and clinical problems. Rinsho Shinkeigaku. 2017;57:716-22.

15 Yeh OL, Francis CE. Ipilimumab-associated bilateral optic neuropathy. J Neuroophthalmol. 2015;35:144-7.

16 Wilson MA, Guld K, Galetta S, Walsh RD, Kharlip J, Tamhankar M, et al. Acute visual loss after ipilimumab treatment for metastatic melanoma. J Immunother Cancer. 2016;4:66.

17 Mori S, Kurimoto T, Ueda K, Enomoto H, Sakamoto M, Keshi Y, et al. Optic neuritis possibly induced by antiPD-L1 antibody treatment in a patient with non-small cell lung carcinoma. Case Rep Ophthalmol. 2018;9: 348-56. 